\title{
MICROSTRUCTURE AND LOCAL TEXTURE IN HOT ROLLED ALUMINUM
}

\author{
H. WEILAND AND J.R. HIRSCH \\ Alcoa Laboratories, Alcoa Center, PA 15069, USA
}

\begin{abstract}
Local textures have been determined in the deformed and partially recrystallized substructure of a hot rolled commercial aluminum alloy by measuring individual grain orientations in a TEM. These local textures were compared to the global textures of the bulk material obtained by conventional X-ray diffraction techniques. Cube oriented nuclei were analyzed and two mechanisms for the development of strong cube textures of hot rolled and subsequently cold rolled and recrystallized aluminum sheet are discussed. These mechanisms are the uniform stability of cube oriented grains during rolling and the generation of cube oriented nuclei by shear strains.
\end{abstract}

\section{EXPERIMENTAL PROCEDURE}

Wedge shaped samples of an aluminum alloy ( $3.5 \mathrm{wt.}-\% \mathrm{Mg}, 0.3 \mathrm{wt} .-\% \mathrm{Mn}$ ) were hot rolled $83 \%$ at $480^{\circ} \mathrm{C}$ and immediately quenched. The material developed a partially recrystallized and inhomogeneous deformed microstructure. The surface was fully recrystallized due to the effects of roll quenching and reheating, and the effects of surface friction. The center showed a deformation substructure which consisted of cells/grains elongated in the rolling direction and different types of deformation bands. In addition, a few grains in the early stages of recrystallization were present at the center. These have been investigated in detail to determine their origin and their orientation relationship to the surrounding matrix.

Bulk textures of the fully recrystallized and of the deformed material were determined from complete pole figures, obtained by X-ray diffraction using a combination of transmission and reflection methods. Local textures were determined by measuring individual grain orientations in a transmission electron microscope (TEM). The orientations were acquired by the on-line evaluation of Kikuchi patterns ${ }^{1}$, obtained by convergent beam electron diffraction. Textures were analyzed using orientation distribution functions (ODFs), which for single orientation data were calculated by superimposing a gaussian shaped peak on each individual orientation.

\section{RESULTS AND DISCUSSION}

The bulk texture of the undeformed, fully recrystallized material consists of a weak cube texture with a scattering of orientations rotated around the normal direction (Fig.1a). During hot rolling, the typical deformation texture of fcc-metals (Fig. 1b-e), which consists of a characteristic orientation tube $(\beta \text {-fibre })^{2}$, is formed . The maximum of the 
$\beta$-fibre is located at the position of the brass component, $\{011\}<211>$, which increases with the degree of deformation. Additionally, the cube texture increases with the degree of hot reduction. Therefore, the presence of cube oriented grains before and after hot deformation raises the question whether cube grains remain from the starting (recrystallized) material or if they are created during hot rolling.

a)

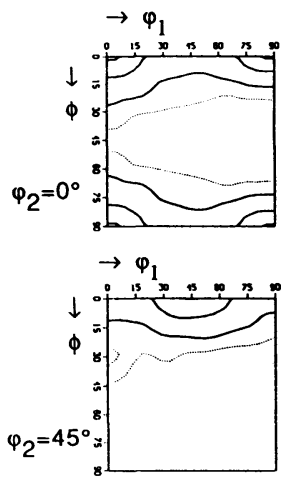

$f_{\max }=6.3$ b)

$\rightarrow \varphi_{1}$

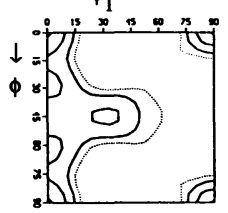

$\rightarrow \varphi_{1}$

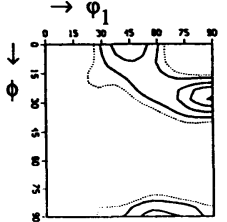

Cont c)

$\rightarrow \varphi_{1}$

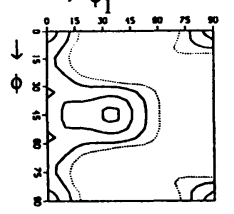

$\rightarrow \varphi_{1}$

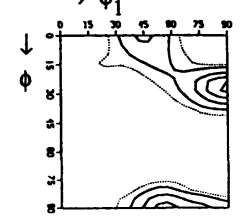

$f \max =9.6$ d)
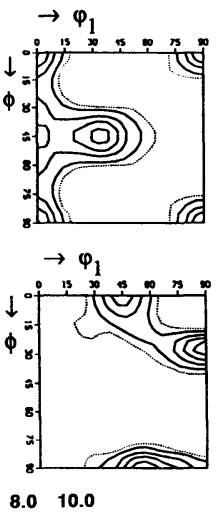

$f_{\max }=11.1$ e)

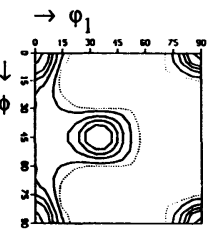

$\rightarrow \varphi_{1}$

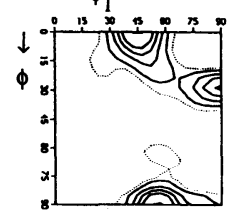

$f_{\max }=14.5$

Figure 1 ODF of the bulk material a) recrystallized and b-e) after different degrees of deformation: b) $54 \%$ c) $72 \%$ d) $79 \%$ e) $83 \%$, measured by X-ray diffraction.

In order to study the recrystallization nuclei, the orientations of those grains, which clearly were in the early stages of recrystallization (figure 2 , marked with *), were measured. These grains are enlarged subgrains, for which the stored energy per volume is smaller than in the surrounding matrix. Commonly, these recrystallization nuclei appeared in groups of 2 or 3 nuclei; isolated nuclei were rare. About 20 vol-\% of the nuclei were cube oriented and the remainder were essentially randomly distributed orientations (Figure 3). The cube proportion of nuclei is the same as the volume fraction of the cube component in the fully recrystallized material (Figure 1a). This suggests that

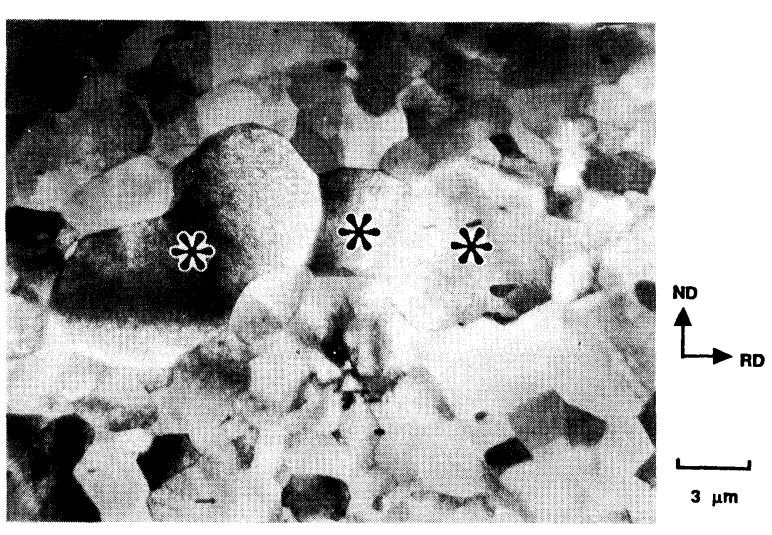

Figure 2 Typical microstructure with recrystallized grains (marked with*). They are cube oriented.

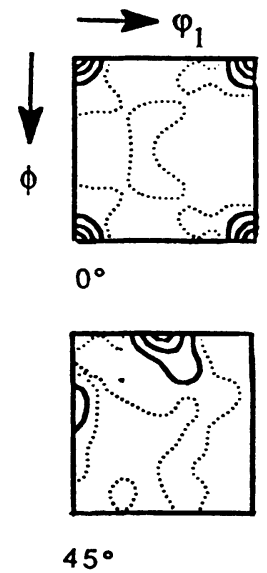

Figure 3 ODF of 50 recrystallization nuclei (TEM measurement). $\varphi_{2}=0^{\circ}$ and $\varphi_{2}=45^{\circ}$ 
the orientations of the nuclei already show the main characteristics of the final texture of the fully recrystallized material, although the statistics for the measured data of 50 grains are limited.

Local areas of the microstructure having predominantly a cube texture were characterized further by means of local texture analysis. Figure 4a shows the microstructure of an analyzed region (area A), which consists of a deformation substructure with subgrains less than $1 \mu \mathrm{m}$ in size. The orientations present are mainly the cube orientation together with a spread towards its rotation around the rolling direction (RD), and orientations near the brass texture component (Figure 4b). These are the main features in the global ODF (Figure 1e).
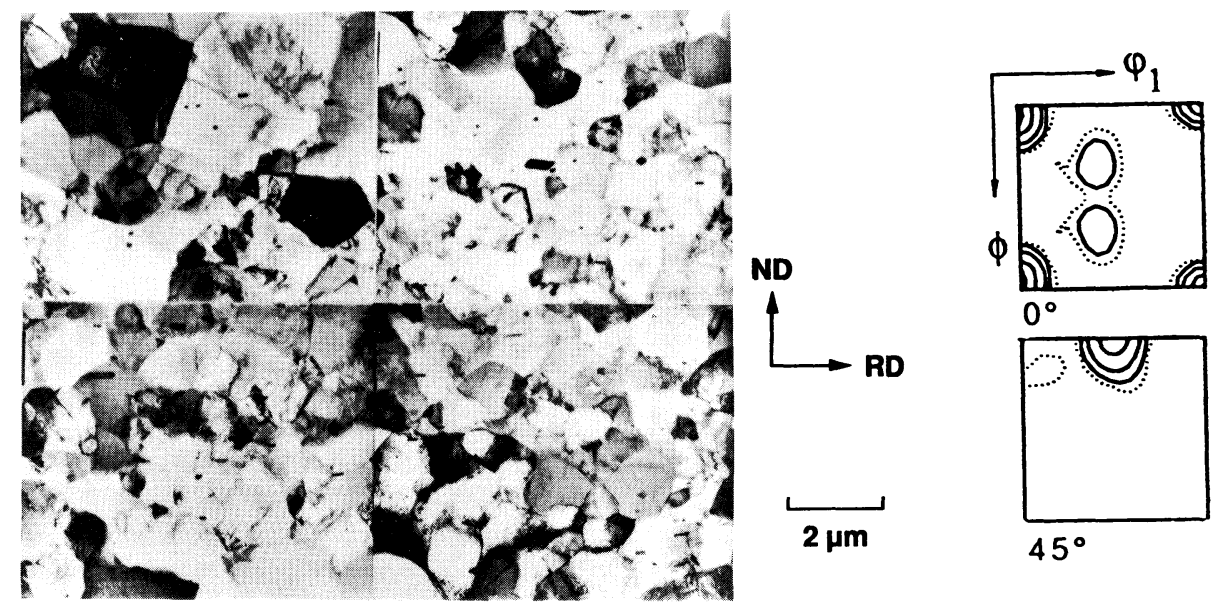

Figure $4 a$ Microstructure of area $A$.

Figure $4 b$ ODF of area $A$ from individual orientation measurements in the TEM.

In order to link individual orientations to the microstructure, the measured orientations were categorized into the ideal components present in the local texture such as cube, brass, $\mathrm{S}$, and the random component. The cube orientations were subdivided further into two groups, one containing those orientations which were misoriented less than $10^{\circ}$ from the ideal cube and the other containing misorientations between $10^{\circ}$ and $20^{\circ}$. According to this orientation classification, the subgrains of a schematic microstructure of area A are outlined differently in figure 5a. The area shows a band of cube oriented grains, elongated in the rolling direction and surrounded by near-cube oriented grains, rotated away from the cube orientation around $R D$. This indicates that before hot rolling a large cube grain was present in the microstructure, which was mostly stable during deformation. At its periphery it has started to rotate around RD away from its initial orientation, as can be predicted with Taylor type models ${ }^{3}$. The remaining subgrains in this area are smaller in size and their orientations are less grouped than the cube oriented ones. These were probably randomly oriented grains which rotated towards the stable deformation texture components in many different ways. In order to be compatible with the surrounding grains, they might have disintegrated into small, highly misoriented subgrains with rolling texture orientations. This seems not to be the case for the cube oriented grains. These have an advantage over other orientations during the following subgrain growth because they rapidly can form significantly larger grains by subgrain coalescence. 
These effects can be illustrated by a different representation of the orientations than in figure 5a, where the orientations were classified only in an approximate manner. A more precise linkage to the microstructure can be achieved by representing the individual orientations as Rodrigues vectors. The length of the vector corresponds to the misorientation angle, while its direction in space corresponds to the rotation axis. In Figure 5b the Rodrigues vectors are superimposed on the microstructure of area $A$ at the center of the corresponding subgrains (for more details on this representation see ref. 4). In the perspective representation of figure $5 \mathrm{~b}$, each vector represents the misorientation with respect to the ideal cube orientation.

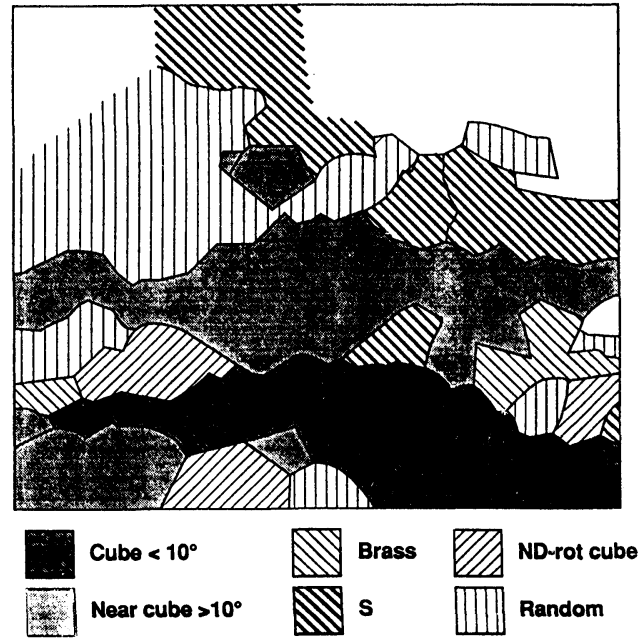

Figure 5a Schematic microstructure of area A. Orientations of individual grains are classified into the typical texture components of aluminum and are outlined differently.

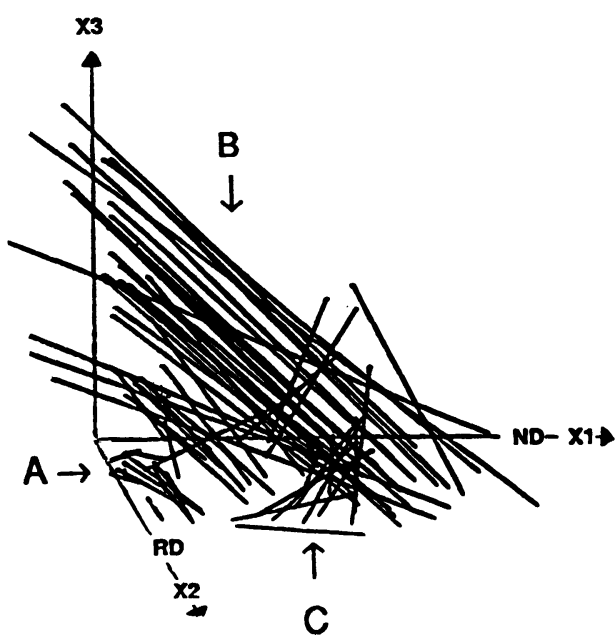

Figure $5 b$ Perspective view of the Rodrigues vectors of area $A$, superimposed on the microstructure. $X 1, X 2$ and $X 3$ are coordinates of the Rodrigues space. $X 1$ and ND are parallel, X2 and RD are parallel.

A group of small vectors (figure $5 \mathrm{~b}$, marked as $\mathrm{A}$ ) represents the cube oriented subgrains. With increasing distance in ND, the length of the vectors increases up to the bundle of longer vectors (marked as B), which belongs to the RD rotated cube grains. The direction of all vectors in A and B is almost constant, which shows that a near cube oriented grain is continually rotating around a constant rotation axis (indicated by the constant direction of the Rodrigues vectors). The amount of rotation (length of the vectors) at the periphery of the initial grain is higher than in its center. Another feature of interest is a set of small vectors in the foreground of figure 5b (marked as C) showing a spiral pattern of rolling type orientations. These vectors represent local rotations probably within one initial grain, generating a set of different orientations during deformation. This indicates that a single grain orientation does not necessarily rotate homogeneously into one deformation orientation. It obviously has used different rotation paths and disintegrated into several subgrains with different orientations, probably in order to maintain compatible strain with its neighborhood.

Another type of local texture was observed (area B, figure 6a). Similar to area A, this type of microstructure consists of a deformation substructure, with subgrains elongated in the rolling direction. The orientations present here are mainly ND rotated cube with a spread towards the ideal cube component (figure 6b). 

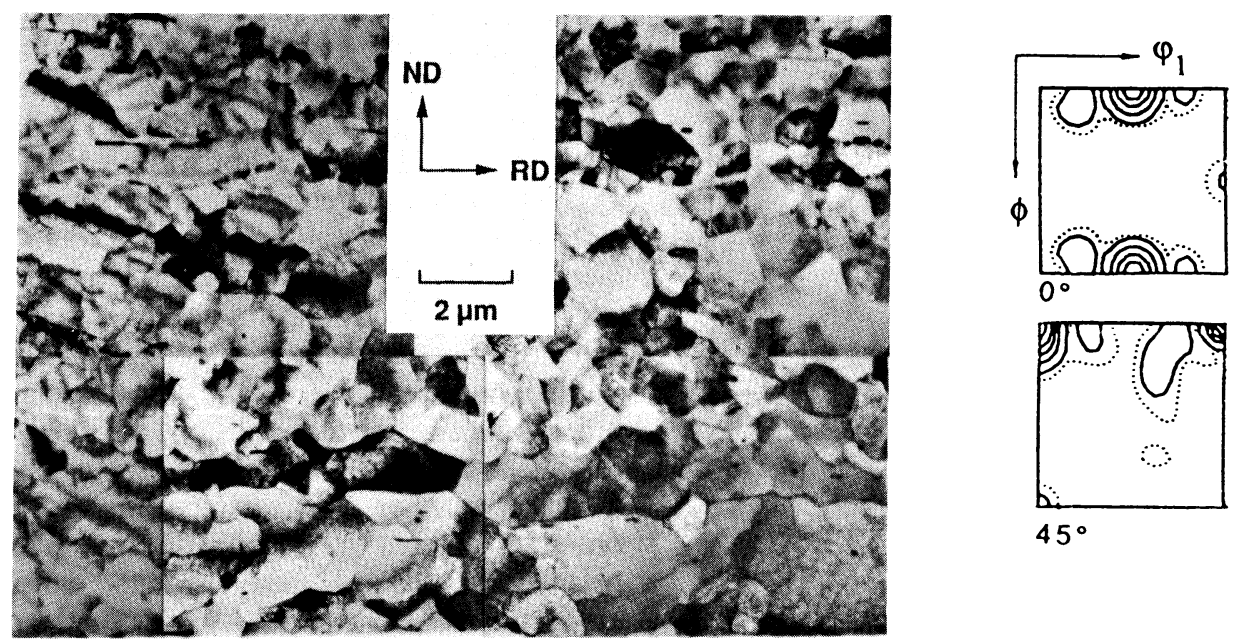

Figure $6 a$ Microstructure of area $B$.

Figure $6 b$ ODF from individual orientation measurements in the TEM of area $B$.

In Figure $6 \mathrm{c}$ the measured orientations again were classified into ideal texture components and outlined separately in the schematic microstructure, as described above. The orientations near the cube component as well as the orientations near the ND rotated cube component each were divided into two classes: a class with a misorientation from the ideal orientation of less than $15^{\circ}$, and a class with a misorientation between $15^{\circ}$ and $25^{\circ}$. This representation of both microstructure and orientations reveals a wide band of grains with the ND rotated cube orientation, elongated in the rolling direction, showing subgrains, which were large relative to those in the remaining area of this microstructure. Together with a second row of grains showing the same orientation, subgrains are enclosed with orientations continually rotating around the normal direction from the ND rotated cube, $\{001\}<110\rangle$, towards the ideal cube.

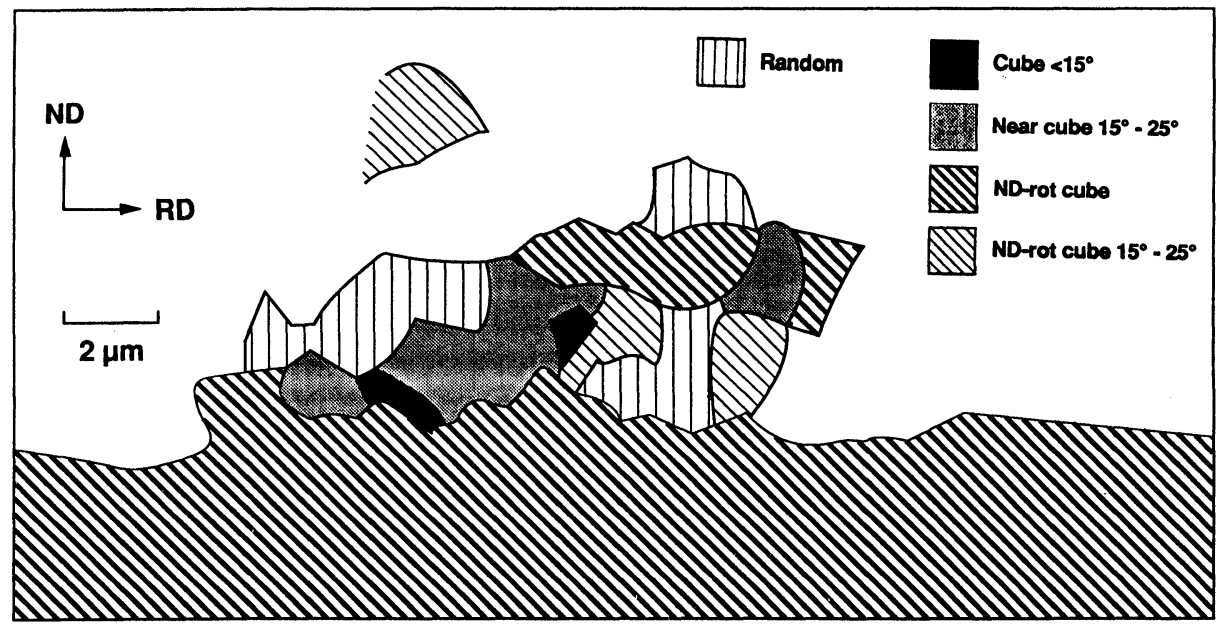

Figure $6 c$ Schematic microstructure of area B. Individual grain orientations are classified into the ideal texture components present in the material and are outlined differently. 


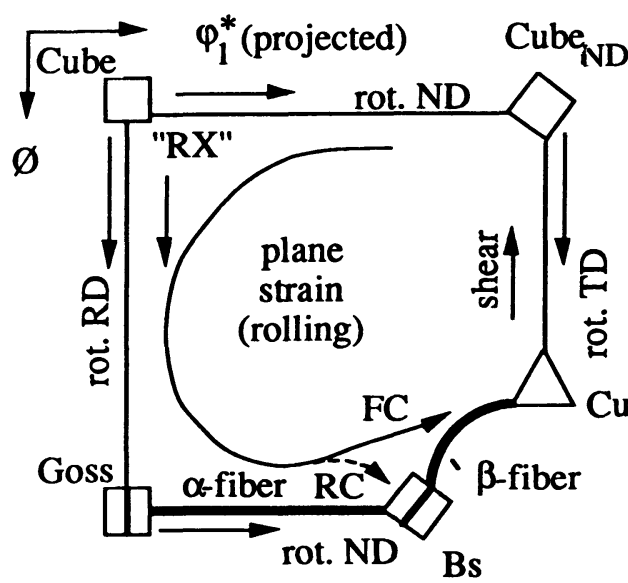

Figure 7 Schematic plot of orientation path of cube orientations in plane strain deformation, projected into the $\varphi_{2}=0^{\circ}$ section $\left(\varphi_{1}+\varphi_{2}=\right.$ const. $)$.

$F C=$ full constraints, $R C=$ relaxed constraints, $R X=$ recrystallization
This local texture indicates a different mechanism for the generation of new cube oriented nuclei. The ND rotated cube is a feature of the global texture after recrystallization as well as after hot rolling (Fig. 1). Hot rolling, in general, and the deformation process in this particular experiment were very inhomogeneous. Due to the increased friction at high temperature and the high reduction of the wedge shaped sample, hot rolling affects the deformation process because surface layers of the material stick to the mill, causing shearing. As discussed earlier 3 , shearing transforms orientations from the $\beta$-fiber (deformation orientations) towards the ND rotated cube component (Figure 7). With further (homogeneous) cold rolling, these orientations rotate towards the cube component and form cube nucleation sites by a Dillamore-Katoh mechanism 5 . In this way, new cube oriented nuclei can be produced during deformation.

\section{CONCLUSIONS}

These local texture studies provide insight into deformation and recrystallization mechanisms during the hot rolling of aluminum alloys. All the orientations which determine the texture of the fully recrystallized material are already present in the early stages of recrystallization. Because cube oriented grains are relatively stable and deform more uniformly during hot rolling, they can more rapidly form nuclei by subgrain growth and coalescence during subsequent recrystallization compared to subgrains with deformation orientations. Additionally, shearing which generates ND rotated cube orientations can provide new cube nucleation sites during further rolling. These two mechanisms probably are responsible for the preferred cube texture formation during hot rolling and after further cold rolling and annealing of aluminum alloys.

\section{REFERENCES}

1. H. Weiland and R. Schwarzer in "Experimental techniques of texture analysis", ed. by H.J. Bunge, Oberursel (FRG) 1986, 301-314

2. J. R. Hirsch, K. Lücke, Acta Metall., Overview, No. 76, Vol. 36, No.11 (1988), pp. 2863-2927

3. J.R. Hirsch, proc. "Recrystallization '90", Wollongong, Australia, ed. T.Chandra, TMS publ. (1990), p.759

4. H. Weiland, J. Liu and E. Nes, this proceedings

5. I.L. Dillamore and H. Katoh, Metal Science Vol. 8 (1974) 73-83. 